\title{
Vibration Suppression of Beam Structures Traversed by Multiple Moving Loads Using a Damped Absorber
}

\author{
Y.H. Lin \\ Department of Mechanical and Marine Engineering National Taiwan Ocean University \\ C.H. Cho \\ Institute of Maritime Technology National Taiwan Ocean University
}

Follow this and additional works at: https://jmstt.ntou.edu.tw/journal

Part of the Mechanical Engineering Commons

\section{Recommended Citation}

Lin, Y.H. and Cho, C.H. (1993) "Vibration Suppression of Beam Structures Traversed by Multiple Moving Loads Using a Damped Absorber," Journal of Marine Science and Technology. Vol. 1: Iss. 1, Article 7.

DOI: 10.51400/2709-6998.2477

Available at: https://jmstt.ntou.edu.tw/journal/vol1/iss1/7

This Research Article is brought to you for free and open access by Journal of Marine Science and Technology. It has been accepted for inclusion in Journal of Marine Science and Technology by an authorized editor of Journal of Marine Science and Technology. 


\section{Vibration Suppression of Beam Structures Traversed by Multiple Moving Loads Using a Damped Absorber}

\section{Acknowledgements}

The authors are grateful for the financial support of this research bv the National Science Council.

Republic of China under the contract No. NSC 81-0403-019-514 and NSC 82-0209-E-019-028 


\title{
VIBRATION SUPPRESSION OF BEAM STRUCTURES TRAVERSED BY MULTIPLE MOVING LOADS USING A DAMPED ABSORBER
}

\author{
Y.H. Lin \\ Department of Mechanical and Marine Engineering \\ National Taiwan Ocean University \\ C.H. Cho \\ Institute of Maritime Technology \\ National Taiwan Ocean University
}

\begin{abstract}
Key words: multiple moving loads, damped absorber, vibration suppression.
\end{abstract}

\begin{abstract}
This paper deals with the dynamic analysis of a simply supported beam subjected to multiple discrete and distributed moving loads. Resonant vibration is observed for the support beam traversed by multiple discrete moving loads. A practical scheme is applied to reduce the excessive beam vibration to an acceptable range by using a damped absorber attached at the beam center. Lagrange's equation is used to formulated the system governing equation and Laplace transformation is applied to derive the dynamic responses for both the beam and the absorber. Various design parameters, such as the mass ratio, the tuning ratio, and the damping ratio, on the vibration reduction performance of the damped absorber are fully analyzed. By properly tuning the absorber with appropriate parameters, excessive beam vibration can be effectively suppressed with a single damped absorber.
\end{abstract}

\section{INTRODUCTION}

Dynamic analysis for structures subjected to moving loads has been a research topic for well over a century. The interest for the study of this problem originated for design of railroad bridges and highway structures [1-2]. Today, the analysis of moving load problem is applicable for various engineering applications such as high speed precision drilling [3], turning [4], workpiece transportation, and fluid flow induced vibration [5-7], etc.

When structures exhibit excessive vibration due to external excitation forces, vibration absorbers have been used extensively for vibration reduction to an acceptable range. Frahm [8] invented a dynamic vibration absorber for lumped parameter systems. Den Hartog [9] refined the theory which includes the damping in the absorber design. Young [10] was the first one to consider the application of lumped parameter absorbers to beams. Optimal design for general beam systems subjected to a point force with the use of damped vibration absorber has also been reported[11].

In this paper, the dynamic characteristics of a simply supported beam traversed by multiple discrete and distributed loads will first be analyzed to understand the system's behavior. Application of a damped absorber is proposed to suppress the excessive vibration of the support beam. The effects of the various design parameters of the absorber will be examined to facilitate efficient engineering design. To the best of the authors' knowledge, the study of this practical scheme for vibration suppression of support beams subjected to multiple moving loads by using a damped vibration absorber has never been reported in the literature and requires in depth analysis to explore its applicability. 


\section{EQUATION OF MOTION}

The equation of motion for a Bernoulli-Euler beam under a general forcing function can be written as

$$
E I \frac{\partial^{4} y}{\partial x^{4}}+\rho A \frac{\partial^{2} y}{\partial t^{2}}=f(x, t)
$$

The boundary conditions for a simply supported beam discussed here are

$$
\begin{aligned}
y(0, t)=y(L, t) & =0 \\
\left.\frac{\partial^{2} y}{\partial x^{2}}\right|_{x=0}=\left.\frac{\partial^{2} y}{\partial x^{2}}\right|_{x=L} & =0
\end{aligned}
$$

and the mode shapes can be written as

$$
\phi_{n}(x)=\sin \frac{n \pi x}{L}
$$

The external forces considered here are multiple concentrated moving loads traveling on the support beam, as shown in Figure 1, and can be represented by Dirac delta function as $F \delta(x-v t)$, which can be expressed using the Fourier sine series expansion, as depicted in Figure 2:

$$
\begin{aligned}
f(x, t) & =\frac{2 F}{L} \sum_{n=1}^{\infty} \sum_{\gamma=1}^{\rho} \sin \frac{n \pi}{\tau}\left(t-\frac{d}{L} \gamma \tau+\tau\right) \\
& {\left[u\left(t-\frac{d}{L} \gamma \tau+\tau\right)-u\left(t-\frac{d}{L} \gamma \tau\right)\right] \sin \frac{n \pi x}{L} }
\end{aligned}
$$

where ' $p$ ' is the number of moving loads, ' $\tau$ ' traveling time for a concentrated load to move from one end of the beam to the other, and ' $d$ ' the distance between two moving loads. For instance as $\frac{d}{L}=1$, it represents

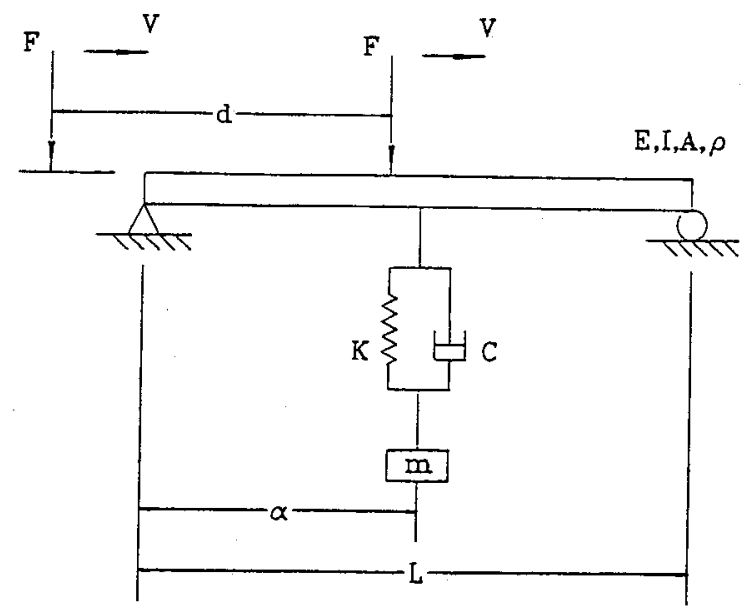

Fig. 1. A simply supported beam with an absorber traversed by multiple moving loads. the case where the distance between two concentrated loads is $L$ and the succeeding load enters the beam at the instant the preceding load leaves the beam span. There will be at most one concentrated force within the beam span at any time for this case. The exact solution for the dynamic response of the support beam due to multiple moving loads as described in Eq. (3) can be shown to be:

$$
\begin{aligned}
& \frac{y_{d}}{y_{s}}=\sum_{n=1}^{\infty} \sum_{\gamma=1}^{p} \frac{96}{\pi^{4} n^{4}\left(1-\frac{a^{2}}{4 n^{4}}\right)}\left\{\left[\sin n \pi\left(\frac{t}{\tau}-\frac{d}{L} \gamma \tau+1\right)\right.\right. \\
&\left.-\frac{a}{2 n} \sin \frac{2 n^{2} \pi}{a}\left(\frac{t}{\tau}-\frac{d}{L} \gamma \tau+1\right)\right] u\left(\frac{t}{\tau}-\frac{d}{L} \gamma \tau+1\right) \\
&-(-1)^{n}\left[\sin n \pi\left(\frac{t}{\tau}-\frac{d}{L} \gamma \tau\right)-\frac{a}{2 n} \sin \frac{2 n^{2} \pi}{a}\right. \\
&\left.\left.\left.\frac{t}{\tau}-\frac{d}{L} \gamma \tau\right)\right] u\left(\frac{t}{\tau}-\frac{d}{L} \gamma \tau\right)\right\} \sin \frac{n \pi x}{L}
\end{aligned}
$$

where ' $y_{d}$ ' is the dynamic response of the beam, ' $y_{s}$ ', maximum static displacement at the beam center due to a single concentrated force, which is $\frac{F L^{3}}{48 E I}$ for the present analysis, and ' $\mathrm{a}$ ' representing $\frac{T_{f}}{\tau}$, in which $T_{f}$ is the fundamental period of the support beam. It can be seen from Eq. (4) that the fundamental mode is the predominant one for contribution to the beam response. Note that in the classical analysis of a support beam traversed by a moving force, resonance speed was observed to occur at $a=2 n$ [12]. From Eq. (4), the same situation applies for the case of multiple moving loads discussed here. However, it has been shown that the above resonance speed will not cause the support beam to vibrate indefinitely [13]. The true resonant vibration for the support beam under multiple moving loads occurs at $\frac{d}{L} / j, j=1,2,3 \ldots$ The purpose of this paper is to study a practical vibration suppression scheme by using a passive damped vibration absorber to reduce excessive vibration of the support beam due to multiple moving loads.

Since vibration of the support beam traversed by multiple moving loads is primarily due to the fundamental mode, the contribution from higher modes can be neglected with sufficient accuracy still being retained. Therefore, the following analysis will consider the first mode only for beam vibration analysis, that is

$$
y_{1}(x, t)=q_{1}(t) \phi(x)
$$

where 


$$
\phi_{1}(x)=\sin \frac{\pi x}{L}
$$

\section{APPLICATION OF A DAMPED VIBRATION ABSORBER}

When the moving speed of the multiple moving forces is at or close to the true resonant speed, the support beam will experience resonance or vibrate excessively, which would make the system unusable or even induce catastrophic structural failure. In this situation, vibration suppression technique needs to be considered to ensure operation accuracy and to avoid material failure when the moving speed and the structural parameters can not be altered due to operation requirements or modification costs. In this study, a practical vibration suppression scheme is proposed and will be analyzed to examine its dynamic characteristics.

The kinetic energy (KE) and potential energy (PE) for a support beam traversed by multiple moving loads with a damped vibration absorber attached, as shown in Figure 1, can be written as

$$
\begin{gathered}
K E=\frac{1}{2} m \dot{q}_{2}^{2}+\frac{1}{2} \rho A \int_{0}^{L} \dot{y}_{1}^{2} d x \\
P E=\frac{1}{2} K\left[y_{1}(\alpha, t)-q_{2}\right]^{2}+\frac{1}{2} E I \int_{0}^{L}\left(\frac{d^{2} y_{1}}{d x^{2}}\right)^{2} d x
\end{gathered}
$$

where $q_{2}$ is the absolute vertical displacement of the absorber. Substitution of Eq. (5) into Eqs. (7) and (8) yields

$$
\begin{gathered}
K E=\frac{1}{2} m \dot{q}_{2}^{2}+\frac{1}{4} \rho \mathrm{AL} \dot{q}_{1}^{2} \\
P E=\frac{1}{2} K\left[\phi_{1}(\alpha) q_{1}-q_{2}\right]^{2}+\frac{1}{4} E I L \beta_{1}^{4} q_{1}^{2}
\end{gathered}
$$

in which $\beta_{1}=\pi / L$. For application of Lagrange's equation, the virtual work done due to the multiple moving loads can be described as

$$
\delta w=\int_{0}^{L} f(x, t) \delta y d x
$$

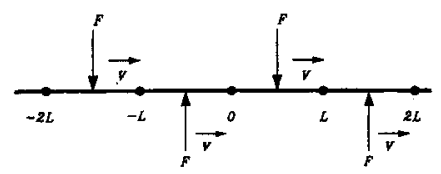

Fig. 2. Fourier since series expansion for a moving load.
The following equation can be obtained by substituting Eq. (3) into Eq. (11)

$$
\begin{aligned}
\delta w & =F \sum_{\gamma=1}^{p} \sin \frac{\pi}{\tau}\left(t-\frac{d}{L} \gamma \tau+\tau\right)\left[u\left(t-\frac{d}{L} \gamma \tau+\tau\right)\right. \\
& \left.-u\left(t-\frac{d}{L} \gamma \tau\right)\right] \delta q_{1}
\end{aligned}
$$

The Lagrange's equation can be written as

$$
\frac{d}{d t}\left(\frac{\partial K E}{\partial \dot{q}_{j}}\right)-\frac{\partial K E}{\partial q_{j}}+\frac{\partial R}{\partial \dot{q}_{j}}+\frac{\partial P E}{\partial q_{j}}=Q_{j}, \quad j=1,2
$$

where ' $R$ ' is the Rayleigh's dissipation function, and ' $Q_{j}$ ' the generalized forces. For the system under consideration

$$
\begin{aligned}
& R=\frac{1}{2} c\left[\dot{q}_{1}-\dot{q}_{2}\right]^{2} \\
& \frac{\partial R}{\partial \dot{q}_{1}}=c\left[\dot{q}_{1}-\dot{q}_{2}\right] \\
& \frac{\partial R}{\partial \dot{q}_{2}}=-c\left[\dot{q}_{1}-\dot{q}_{2}\right]
\end{aligned}
$$

in which ' $c$ ' is the viscous damping coefficient. From Eqs. (13 - 16) and Eqs. (9), (10), and (12), the equation of motion for the entire system is

$$
\begin{aligned}
& {\left[\begin{array}{cc}
\frac{1}{2} \rho A L & 0 \\
0 & m
\end{array}\right]\left[\begin{array}{l}
\ddot{q}_{1} \\
\ddot{q}_{2}
\end{array}\right]+\left[\begin{array}{cc}
c & -c \\
-c & c
\end{array}\right]\left[\begin{array}{l}
q_{1} \\
\dot{q}_{2}
\end{array}\right]} \\
& {\left[\begin{array}{cc}
K \phi_{1}^{2}(\alpha)+\frac{1}{2} E I L \beta_{1}^{4} & -K \phi_{1}(\alpha) \\
-K \phi_{1}(\alpha) & K
\end{array}\right]\left[\begin{array}{l}
q_{1} \\
q_{2}
\end{array}\right]} \\
& =\left[\begin{array}{c}
f(t) \\
0
\end{array}\right] .
\end{aligned}
$$

Applying Laplace transformation and Cramer's rule for the above equation, and using dimensionless expression, $Q_{1}(s)$ and $Q_{2}(s)$ can be obtained as

$$
\begin{aligned}
Q_{1}(s)= & \frac{2 F}{E I L \beta_{1}^{4} w_{1}} \frac{\left(0.5 a \frac{s^{2}}{w_{1}^{2}}+a \zeta \frac{s}{w_{1}}+0.5 a T^{2}\right)}{w_{1}^{6}+A_{1} \frac{s^{5}}{w_{1}^{5}}+A_{2} \frac{s^{4}}{w_{1}^{4}}+A_{3} \frac{s^{3}}{w_{1}^{3}}} \\
& \frac{\left(e^{-\left(\frac{d}{L} \gamma \tau-\tau\right) s}+e^{-\frac{d}{L} \gamma \tau s}\right)}{A_{4} \frac{s^{2}}{w_{1}^{2}}+A_{5} \frac{s}{w_{1}}+A_{6}}
\end{aligned}
$$




$$
\begin{aligned}
Q_{2}(s)= & \frac{2 F}{E I L \beta_{1}^{4} w_{1}} \frac{\left(a \zeta \frac{s}{w_{1}}+0.5 a T^{2}\right)}{w_{1}^{6}}+A_{1} \frac{s^{5}}{w_{1}^{5}}+A_{2} \frac{s^{4}}{w_{1}^{4}}+A_{3} \frac{s^{3}}{w_{1}^{3}} \\
& \frac{\left(e^{-\left(\frac{d}{L} \gamma \tau-\tau\right) s}+e^{-\frac{d}{L} \gamma \tau s}\right)}{A_{4} \frac{s^{2}}{w_{1}^{2}}+A_{5} \frac{s}{w_{1}}+A_{6}}
\end{aligned}
$$

in which

$T^{2}=\frac{K}{m} \times \frac{\rho A}{E I \beta_{1}^{4}}$, the tuning ratio

$\mu=\frac{m}{\rho A L}$, the mass ratio

$\zeta=\frac{c}{2 m w_{1}}$, the damping ratio

ing inverse Laplace transformation for Eqs. (21) and (22) and using dimensionless expressions, $\frac{q_{1}(t / \tau, x)}{y_{s}}$ and $\frac{q_{2}(t / \tau)}{y_{s}}$ can be obtained. For $\alpha=\frac{L}{2}$, the dynamic responses at the beam center and the absorber can then be written as

$$
\frac{y_{1}\left(\frac{t}{\tau}, \frac{L}{2}\right)}{y_{s}}=\frac{96}{\pi^{4}} \sum_{\gamma=1}^{p} \sum_{i=1}^{6}\left\{\left|B_{i}\right| e^{R_{e}\left(D_{i}\right) \frac{2 \pi}{a}\left(\frac{t}{\tau}-\frac{d}{L} \gamma+1\right)}\right.
$$

$$
\begin{aligned}
& \cos \left[\operatorname{Im}\left(D_{i}\right) \frac{2 \pi}{a}\left(\frac{t}{\tau}-\frac{d}{L} \gamma+1\right)+\theta_{i}\right] u\left(\frac{t}{\tau}-\frac{d}{L} \gamma+1\right) \\
& +\left|G_{i}\right| e^{R} e^{(H} i^{\frac{2 \pi}{a}\left(\frac{t}{\tau}-\frac{d}{L} \gamma\right)} \cos \left[\operatorname{Im}\left(H_{i}\right) \frac{2 \pi}{a}\left(\frac{t}{\tau}-\frac{d}{L} \gamma\right)\right. \\
& \left.\left.+\theta_{i}\right] u\left(\frac{t}{\tau}-\frac{d}{L} \gamma\right)\right\} \\
& \frac{q_{2}\left(\frac{t}{\tau}\right)}{y_{s}}=\frac{96}{\pi^{4}} \sum_{\gamma=1}^{p} \sum_{i=1}^{6}\left\{\left|G_{i}\right| e^{R_{e}\left(H_{i}\right) \frac{2 \pi}{a}\left(\frac{t}{\tau}-\frac{d}{L} \gamma+1\right)}\right. \\
& \cos \left[\operatorname{lm}\left(H_{i}\right) \frac{2 \pi}{a}\left(\frac{t}{\tau}-\frac{d}{L} \gamma+1\right)+\varphi_{i}\right] u\left(\frac{t}{\tau}-\frac{d}{L} \gamma+1\right)
\end{aligned}
$$

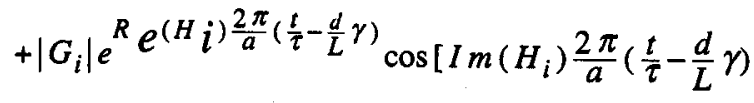

$$
\begin{aligned}
& \left.\left.+\varphi_{i}\right] u\left(\frac{t}{\tau}-\frac{d}{L} \gamma\right)\right\}
\end{aligned}
$$

where $|\bullet|, \operatorname{Re}(\bullet)$, and $\operatorname{Im}(\bullet)$ represent magnitude, real part, and imaginary part of a complex number, respectively.

As the distance, $d$, between two moving loads approaches zero, that is when the support beam is traversed by distributed moving load, the external force can be described by the application of unit step function, $F u(x-v t)$, and can be written as

$$
\begin{gathered}
f(x, t)=\sum_{n=1}^{\infty} \frac{2 w}{n \pi}\left(1-\cos \frac{n \pi v t}{L}\right)[u(t) \\
-u(t-\tau)] \sin \frac{n \pi x}{L}
\end{gathered}
$$

$$
\begin{gathered}
Q_{2}(s)=\frac{2 F}{E I L \beta_{1}^{4}} \sum_{\gamma=1}^{p} \sum_{i=1}^{6} \frac{G_{i} w_{1}}{s-H_{i} w_{1}} \\
{\left[e^{-\left(\frac{d}{L} \gamma \tau-\tau\right) s}+e^{-\frac{d}{L} \gamma \tau s}\right]}
\end{gathered}
$$

by applying Fourier sine series expansion. Substituting Eq. (25) into Eq. (1), the normalized dynamic response of the support beam can be solved:

$$
\frac{y_{d}}{y_{u}}=\frac{768}{5 n^{5} \pi^{5}}\left\{\left[1-\cos \frac{2 n^{2} \pi}{a} \frac{t}{\tau}+\frac{1}{1-0.25 \frac{a^{2}}{n^{2}}}\right.\right.
$$

in which $B_{i}, D_{i}, G_{i}$, and $H_{i}$ are obtained numerically. Note that they may occur as complex numbers. Tak- 


$$
\left(\frac{t}{\tau}-1\right)+\frac{1}{1-0.25 \frac{a^{2}}{n^{2}}}\left(\cos \frac{2 n^{2} \pi}{a}\left(\frac{t}{\tau}-1\right)\right.
$$

$\left.\left.\left.-\cos n \pi\left(\frac{t}{\tau}-1\right)\right)\right] u\left(\frac{t}{\tau}-1\right)\right\} \sin \frac{n \pi x}{L}$

in which $y_{u}=\frac{5 w L^{4}}{384 E I}$, ' $w$ 'being the load per unit length.

For the case of distributed moving load, the virtual work done can be expressed as

$$
\delta_{w}=\frac{\omega L}{\pi}\left(1-\cos \frac{\pi v t}{L}\right)[u(t)-u(t-\tau)] \delta_{q_{1}}
$$

Analogous to the previous development, $Q_{I}(s)$ and $Q_{2}(s)$ can be obtained as

$$
\begin{aligned}
& Q_{1}(s)=\frac{2 \omega}{\pi E I \beta_{1}^{4} \omega_{1}}\left[\frac{1}{\frac{s}{\omega_{1}}}-\frac{\frac{s}{\omega_{1}}}{\left(\frac{s}{\omega_{1}}\right)^{2}+0.25 a^{2}}\right] \\
& \left\{\frac{\left[\left(\frac{s}{\omega_{1}}\right)^{2}+2 \zeta \frac{s}{\omega}+T^{2}\right]}{\left(\frac{s}{\omega_{1}}\right)^{4}+\widetilde{A}_{1}\left(\frac{s}{\omega_{1}}\right)^{3}+\widetilde{A}_{2}\left(\frac{s}{\omega_{1}}\right)^{2}+}\right. \\
& \left.\frac{\left[1+e^{-\tau s}\right]}{\AA_{3}\left(\frac{s}{\omega_{1}}\right)+T^{2}}\right\} \\
& Q_{2}(s)=\frac{2 \omega}{\pi E I \beta_{1}^{4} \omega_{1}}\left[\frac{1}{\frac{s}{\omega_{1}}}-\frac{\frac{s}{\omega_{1}}}{\left(\frac{s}{\omega_{1}}\right)^{2}+0.25 a^{2}}\right] \\
& \left\{\frac{\left[2 \zeta \frac{s}{\omega}+T^{2}\right]}{\left(\frac{s}{\omega_{1}}\right)^{4}+\tilde{A}_{1}\left(\frac{s}{\omega_{1}}\right)^{3}+\tilde{A}_{2}\left(\frac{s}{\omega_{1}}\right)^{2}+}\right. \\
& \left.\frac{\left[1+e^{-\tau s}\right]}{\widetilde{A}_{3}\left(\frac{s}{\omega_{1}}\right)+T^{2}}\right\}
\end{aligned}
$$

where

$\widetilde{A}_{1}=2 \zeta(1+2 \mu)$

$\widetilde{A}_{2}=1+T^{2}+2 \mu T^{2} \phi_{1}^{2}(\alpha)$

$\tilde{A}_{3}=2 \zeta\left[1+2 \mu T^{2}+2 \mu T^{2} \phi_{1}^{2}(\alpha)-4 \mu T^{2} \phi_{1}(\alpha)\right]$

Taking the inverse Laplace transformation, dynamic responses at the beam center and the absorber are obtained:

$\frac{y_{1}\left(\frac{t}{\tau}, \frac{L}{2}\right)}{y_{u}}=\frac{768}{5 \pi^{5}} \sum_{i=1}^{11}\left\{\left|I_{i}\right| e^{R_{e}\left(J_{i}\right) \frac{2 \pi t}{a \tau}} \cos \left[\operatorname{Im}\left(J_{i}\right) \frac{2 \pi t}{a \tau}\right.\right.$

$$
\begin{aligned}
& \left.+\theta_{i}\right] u\left(\frac{t}{\tau}\right)+\left|I_{i}\right| e^{R_{e}\left(J_{i}\right) \frac{2 \pi}{a}\left(\frac{t}{\tau}-1\right)} \\
& \left.\cos \left[I m\left(J_{i}\right) \frac{2 \pi}{a}\left(\frac{t}{\tau}-1\right)+\theta_{i}\right] u\left(\frac{t}{\tau}-1\right)\right\}
\end{aligned}
$$

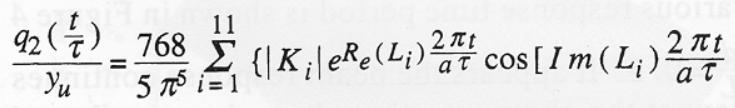

$$
\begin{aligned}
& \left.+\varphi_{i}\right] u\left(\frac{t}{\tau}\right)+\left|K_{i}\right| e^{R_{e}\left(L_{i}\right) \frac{2 \pi}{a}\left(\frac{t}{\tau}-1\right)} \\
& \left.\cos \left[\operatorname{Im}\left(L_{i}\right) \frac{2 \pi}{a}\left(\frac{t}{\tau}-1\right)+\varphi_{i}\right] u\left(\frac{t}{\tau}-1\right)\right\}
\end{aligned}
$$

in which $\theta_{i}=\tan ^{-1} \frac{\operatorname{Im}\left(I_{i}\right)}{\operatorname{Re}\left(I_{i}\right)}, \varphi_{i}=\tan ^{-1} \frac{\operatorname{Im}\left(K_{i}\right)}{\operatorname{Re}\left(K_{i}\right)}$, and $I_{i}$ $J_{i}, K_{i}$, and $L_{i}$ are obtained numerically as discussed previously.

\section{NUMERICAL RESULTS}

Figure 3 illustrates the normalized dynamic response at the beam center versus the moving load speed parameter, $a$, for $\frac{d}{L}=0.5$, which represents the case where there are at most two moving loads within the beam span at any time. The true resonant speeds correspond to $a=\frac{d}{L} / j . \mathrm{j}=1,2,3, \ldots$, at which the new coming load eriters the beam when the beam is going downward and lience the response level keeps grow

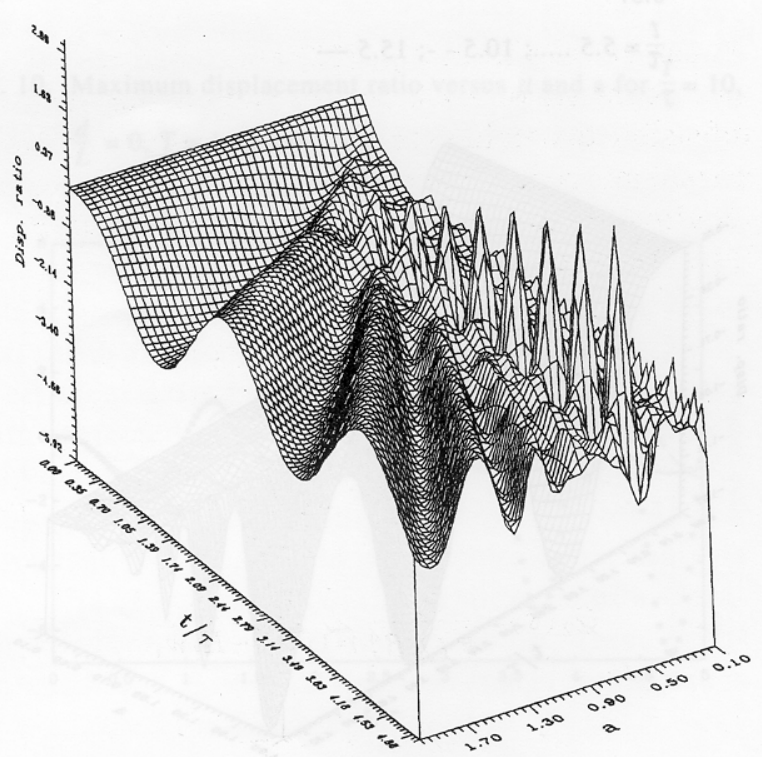

Fig. 3. Dynamic response at the beam center versus moving speed, $\frac{d}{L}=0.5$. 
ing if moving loads continue entering the beam. The maximum displacement ratio, defined as the maximum dynamic response at the beam center over the maximum static displacement at the beam center due to a single force, versus the moving load parameter, $a$, for various response time period is shown in Figure 4 for $\frac{d}{L}=0.5$. It appears the beam response continues to grow at the above mentioned moving speeds and such situation will not occur for moving speed above $a=0.5$, which indicates higher moving speed does not necessarily induce larger dynamic impact on the support beam.

Normalized dynamic response at the beam center versus the moving speed parameter, $a$, for $\frac{d}{L}=0$, i.e. the distributed moving load case, is depicted in Figure 5. Note that as the moving speed is slow, the beam response resembles the static case, as would be

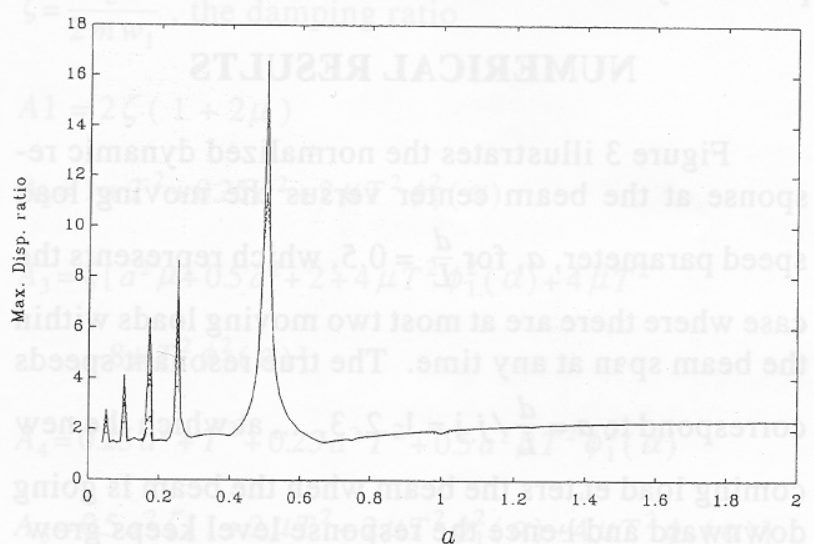

Fig. 4. Maximum displacement ratio versus moving speed, $\frac{d}{L}=$ 0.5 .

$\frac{t}{\tau}=5.5 \ldots \ldots ; 10.5--; 15.5-$

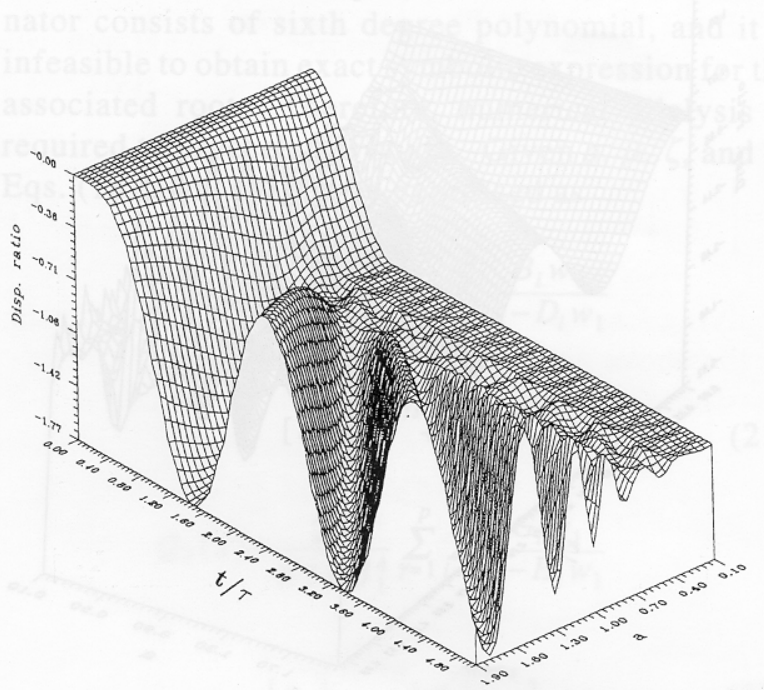

Fig, 5. Dynamic response at the beam center versus moving speed, $\frac{d}{L}=0$ (distributed load). expected. As the moving speed increases, larger beam dynamic response can be observed. However, unlike the discrete moving load case, no resonant speed exists for the distributed moving load problem. Figure 6 shows the maximum displacement ratio versus the moving speed parameter, $a$, for various response time period. Note that the beam response does not increase as the response time period increases, which is different to the case of multiple discrete moving load problem. As the moving speed further increases, the beam response reaches a constant maximum displacement ratio, which is two for this analysis. This resembles the case with sudden apply of a load on a dynamic system and the maximum dynamic response is twice that of static response.

Effectiveness of the application of a damped vibration absorber to suppress the excessive beam vibration is depicted in Figures 7 and 8, with the significance of the design parameters being examined. It appears the tuning ratio parameter, $\mathrm{T}$, around unity gives the most favorable results for vibration reduction of the support beam. With the increase of the mass ratio, $\mu$, the beam vibration decreases. However, for $\mu$ approximately above 0.32 , its effectiveness approaches the plateau, which indicates limited improvement with even higher mass ratio. Note that the mass ratio should be kept minimal to reduce the total system weight. With $\frac{d}{L}=05$, it has been shown that the true resonant speeds are at $a=0.5 / \mathrm{j}, \mathrm{j}=1,2$, .... Figure 8 shows that with the application of the vibration absorber, the corresponding beam vibration is effectively suppressed at those speeds, and that with the increase of the mass ratio, the effective range of vibration suppression around those speeds widened, which indicates broader usable 'speed band' for higher mass ratio.

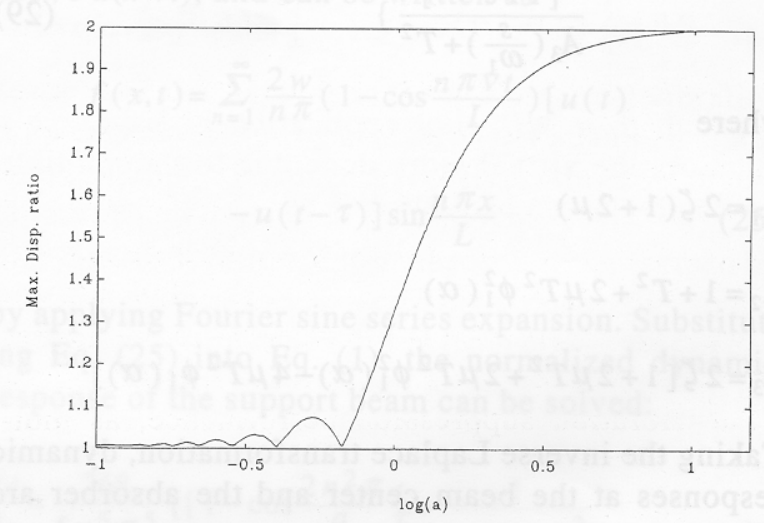

Fig. 6. Maximum displacement ratio versus moving speed, $\frac{d}{L}=0$.
$\frac{t}{\tau}=10 \ldots . ; 20--; 30-$ 


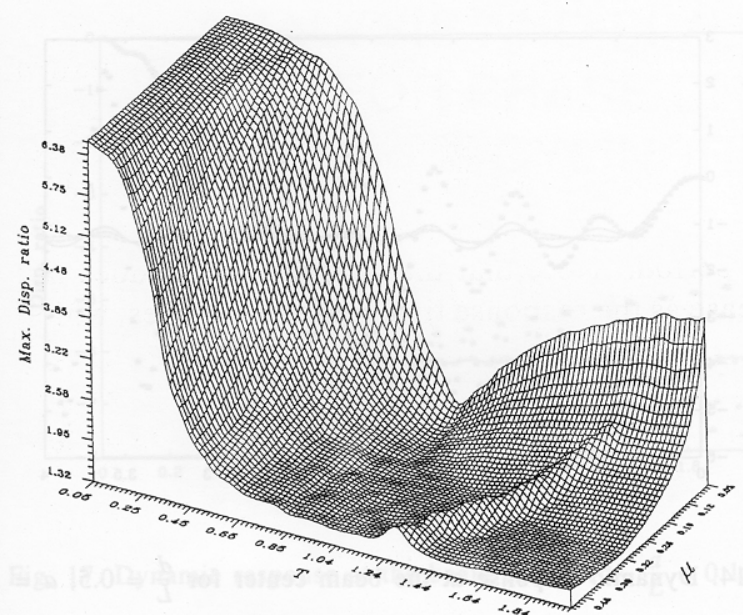

Fig. 7. Maximum displacement ratio versus $\mu$ and $\mathrm{T}$ for $\frac{t}{\tau}=5.5$, $\frac{d}{L}=0.5, a=0.5, \zeta=0$.

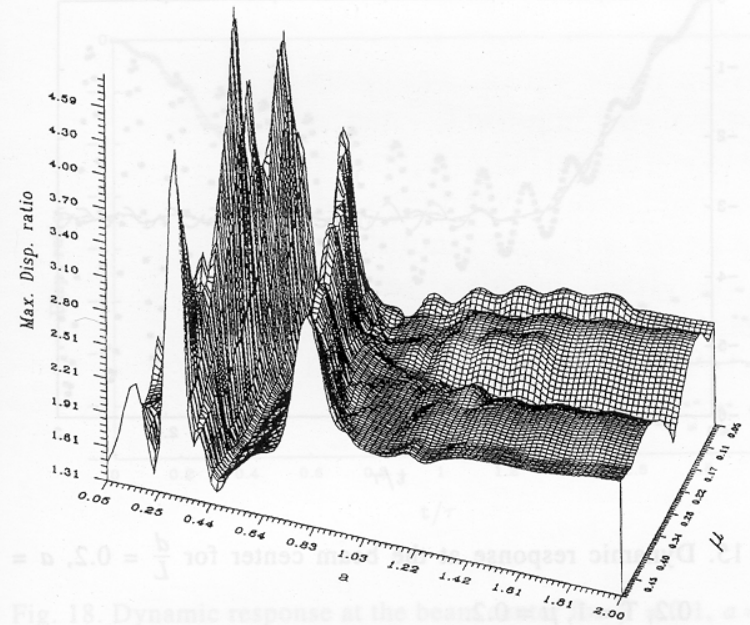

Fig. 8. Maximum displacement ratio versus $\chi$ and a for $\frac{t}{\tau}=5.5$, $\frac{d}{L}=0.5, \mathrm{~T}=1, \zeta=0$.

As for the case of distributed moving load, Figures 9 and 10 illustrate the effects of the various design parameters. It turns out the use of vibration absorber for the distributed moving load problem is not as effective as that for multiple discrete moving load case, since the former does not have the problem of resonant vibration for any moving speed. For higher moving speed, the use of mass ratio, $\mu=0.25$ gives better vibration suppression performance, as indicated in Figure 10.

Figure 11 shows the normalized dynamic response at the beam center for $\frac{d}{L}=1, a=1, T=1$, and $\mu=0.2$, with various damping ratios. The support beam exhibits resonant vibration without the application of absorber. The case with $\zeta=0.1$ yields best performance among the three different damping val-

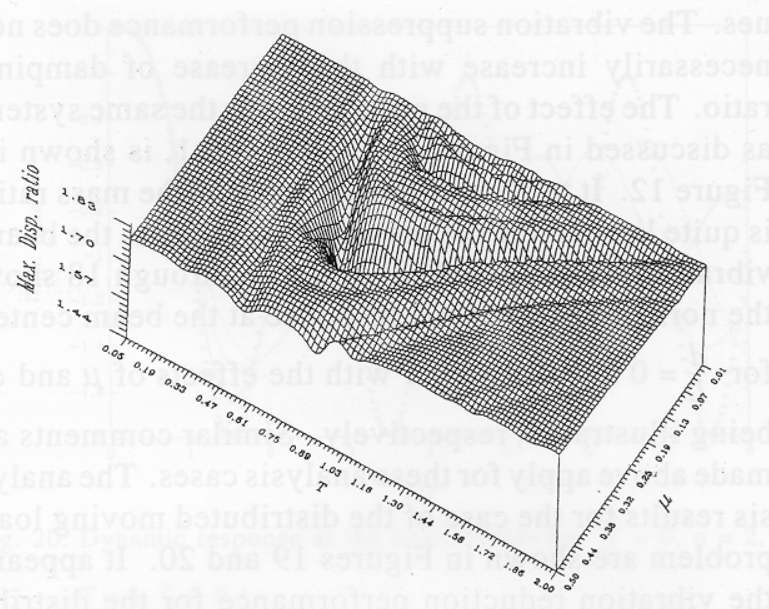

Fig. 9. Maximum displacement ratio versus $\mu$ and $\mathrm{T}$ for $\frac{t}{\tau}=10$, $\frac{d}{L}=0, a=2, \zeta=0$.

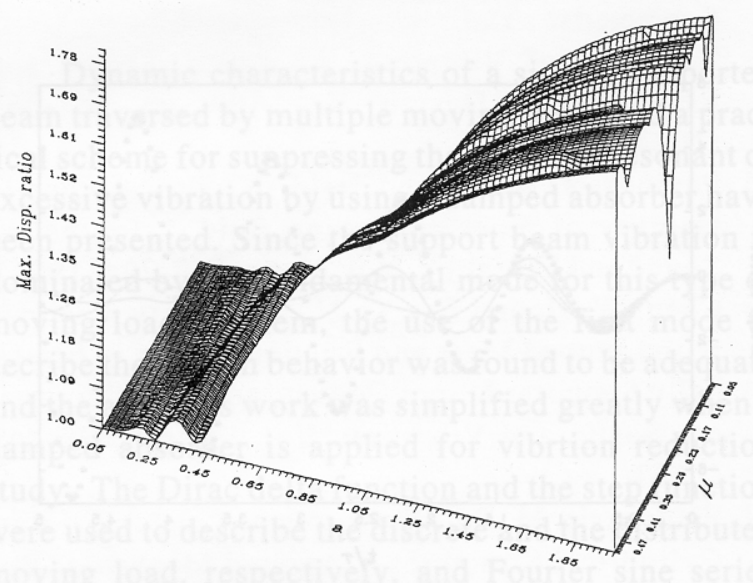

Fig. 10. Maximum displacement ratio versus $\mu$ and a for $\frac{t}{\tau}=10$, $\frac{d}{L}=0, T=1, \zeta=0$.

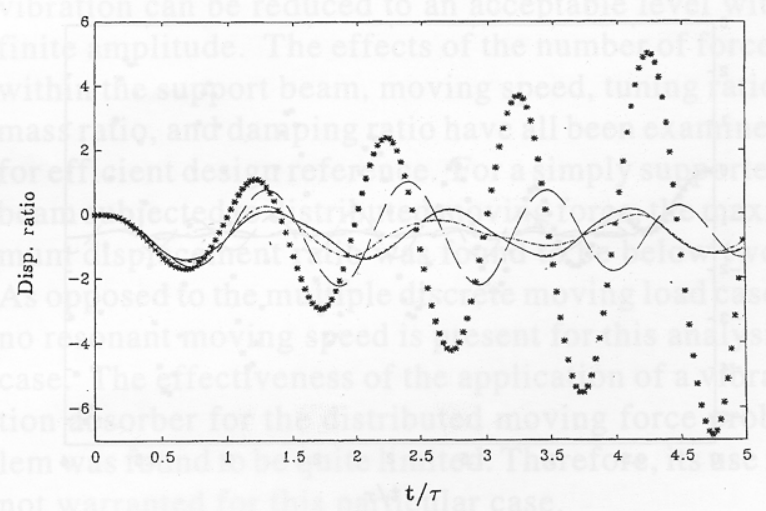

Fig. 11. Dynamic response at the beam center for $\frac{d}{L}=1, a=1$,

$\mathrm{T}=1, \mathrm{~T}=1,=0.2$.

$\zeta=0-; 0.01--; 0.1-.-.-; 1--;$

without absorber $* * *$ 
ues. The vibration suppression performance does not necessarily increase with the increase of damping ratio. The effect of the mass ratio for the same system as discussed in Figure 11, with $\zeta=0.1$, is shown in Figure 12. It turns out the influence of the mass ratio is quite limited for the present case as far as the beam vibration is concerned. Figures 13 through 18 show the normalized dynamic response at the beam center for $\frac{d}{L}=05,0.2$, and 0.1 with the effects of $\mu$ and $\zeta$ being illustrated, respectively. Similar comments as made above apply for these analysis cases. The analysis results for the case of the distributed moving load problem are shown in Figures 19 and 20. It appears the vibration reduction performance for the distributed moving load problem is not as effective as that for the multiple discrete moving load problem, hence the use of vibration absorber for this particular case is not warranted.

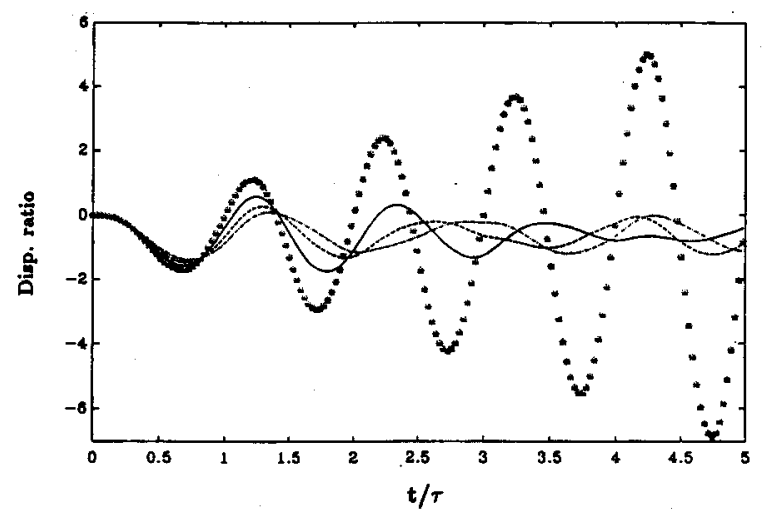

Fig. 12. Dynamic response at the beam center for $\frac{d}{L}=1, a=1, T$ $=1, \zeta=0.1$.

$\mu=0.1-; 0.2--; 0.3-.-.-$; without absorber ***

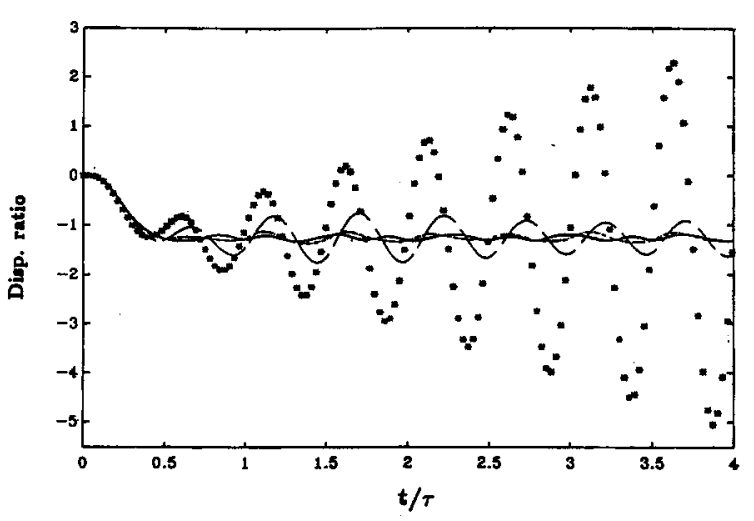

Fig. 13. Dynamic response at the beam center for $\frac{d}{L}=0.5, a=$ $0.5, \mathrm{~T}=1, \mu=0.2$. $\zeta=0 \longrightarrow ; 0.01 \longrightarrow ; 0.1-.-. ; 1-;$ without absorber ***

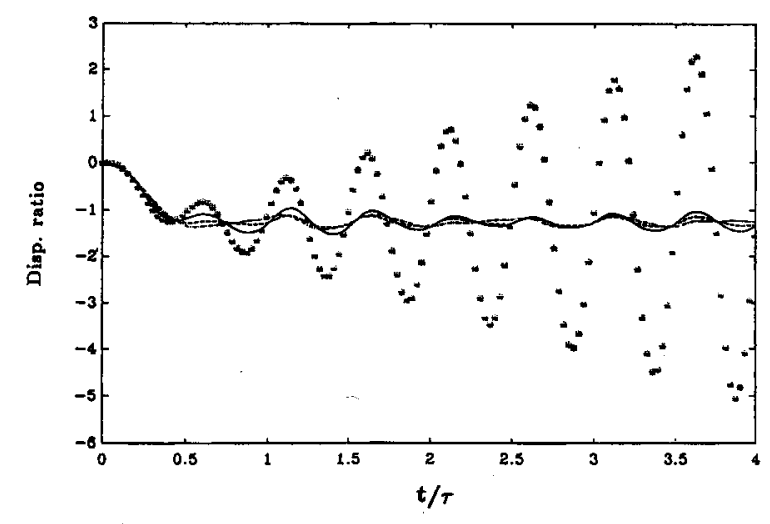

Fig. 14. Dynamic response at the beam center for $\frac{d}{L}=0.5, a=$ $0.5, \mathrm{~T}=1, \zeta=0.1$. $\mu=0.1-; 0.2--; 0.3-.$, . -; without absorber ***

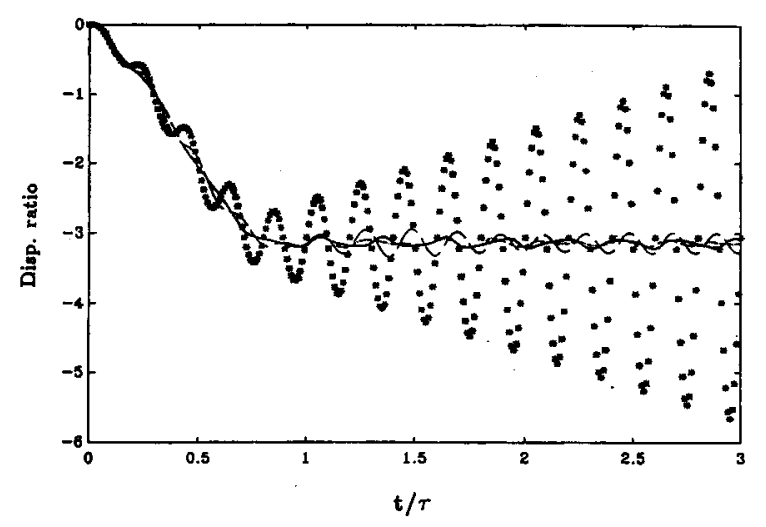

Fig. 15. Dynamic response at the beam center for $\frac{d}{L}=0.2, a=$ $0.2, \mathrm{~T}=1, \mu=0.2$. $\zeta=0-; 0.01-; 0.1 . .-.-; 1-;$ without absorber ***

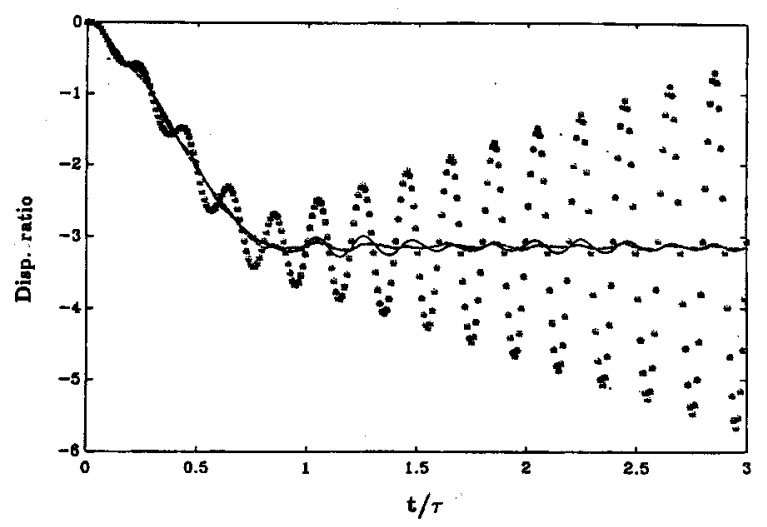

Fig. 16. Dynamic response at the beam center for $\frac{d}{L}=0.2, a=$ $0.2, \mathrm{~T}=1, \zeta=0.1$. $\mu=0.1-; 0.2-\div 0.3-$ - - . -; without absorber *** 


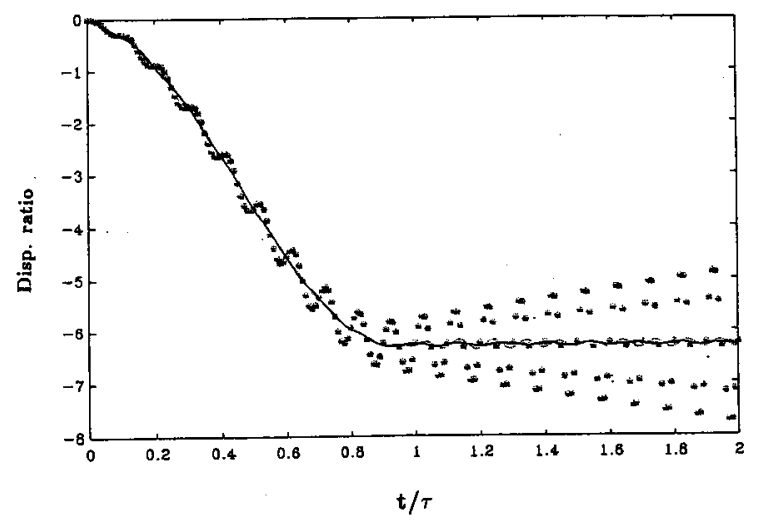

Fig. 17. Dynamic response at the beam center for $\frac{d}{L}=0.1, a=$

$0.1, \mathrm{~T}=1, \mu=0.2$.

$\zeta=0-; 0.01--; 0.1-.-.-; . .$. ;

without absorber ***

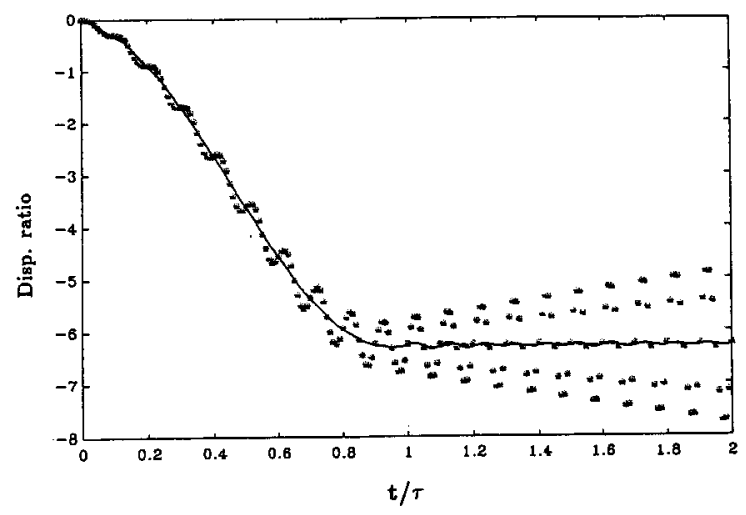

Fig. 18. Dynamic response at the beam center for $\frac{d}{L}=0.1, a=01$,

$\mathrm{T}=1, \mu=0.1$.

$\mu=0.1-; 0.2--; 0.3-.-.-$; without absorber $* * *$

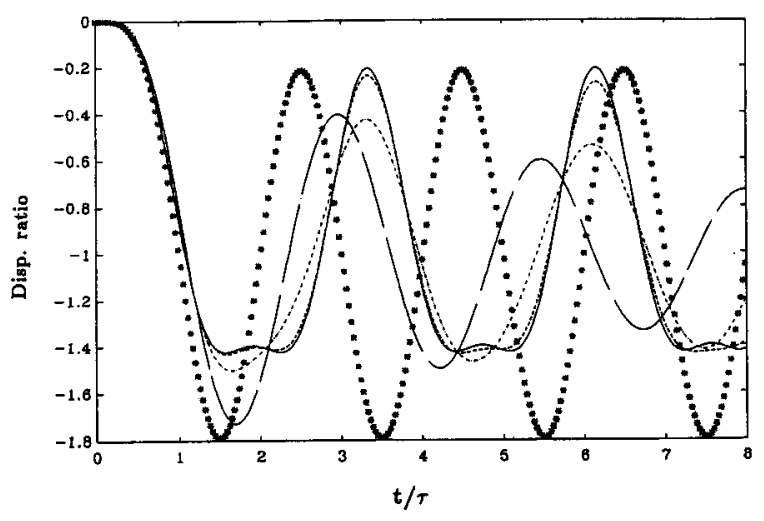

Fig. 19. Dynamic response at the beam center for $\frac{d}{L}=0, a=2$,

$\mathrm{T}=1, \mu=0.25$.

$\zeta=0-; 0.01--; 0.1-.-.-; 1--;$ without absorber

$* * *$

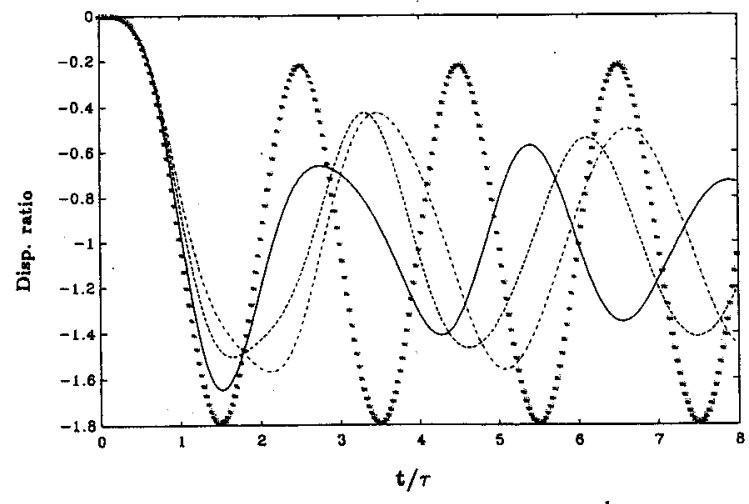

Fig. 20. Dynamic response at the beam center for $\frac{d}{L}=0, a=2$,

$$
\begin{aligned}
& \mathrm{T}=1, \zeta=0.1 . \\
& \mu=0.1-; 0.25--; 0.4-.-.-; \text { without absorber } * * *
\end{aligned}
$$

\section{CONCLUSIONS}

Dynamic characteristics of a simply supported beam traversed by multiple moving loads and a practical scheme for suppressing the resulting resonant or excessive vibration by using a damped absorber have been presented. Since the support beam vibration is dominated by the fundamental mode for this type of moving load problem, the use of the first mode to decribe the system behavior was found to be adequate and the amalysis work was simplified greatly when a damped absorber is applied for vibrtion reduction study. The Dirac delta function and the step function were used to describe the discrete and the distributed moving load, respectively, and Fourier sine series expansion was then applied to facilitate further analysis. In this paper, it was found the use of a damped vibration absorber is effective for suppressing the excessive support beam vibration for the multiple discrete moving load problem. Original resonant vibration can be reduced to an acceptable level with finite amplitude. The effects of the number of forces within the support beam, moving speed, tuning ratio, mass ratio, and damping ratio have all been examined for efficient design reference. For a simply supported beam subjected to distributed moving force, the maximum displacement ratio was found to be below two. As opposed to the multiple discrete moving load case, no resonant moving speed is present for this analysis case. The effectiveness of the application of a vibration absorber for the distributed moving force problem was found to be quite limited. Therefore, its use is not warranted for this particular case.

\section{ACKNOWLEDGEMENT}

The authors are grateful for the financial support of this research bv the National Science Council. 
Republic of China under the contract No. NSC 810403-019-514 and NSC 82-0209-E-019-028 .

\section{REFERENCES}

1. Stokes, G. G., “ Discussions of a Differential Equation Relating to the Breaking of Railway Bridges, "Mathematical and Physical Papers, Vol. 2, pp. 178-220 (1883).

2. Inglis, C. E., A Mathematical Treatise on Vibration in Railway Bridges. Cambridge University Press (1934).

3. Lin, Y.-H., Trethewey, M. W., Reed, H. M., Shawley, J. D., and Sager, S. J., " Dynamic Modeling and Analysis of a High Speed Precision Drilling Machine," Journal of Vibration and Acoustics, Vol.112, No. 3, pp. 355-365 (1990).

4. Katz, R., Lee, C. W. Vlsoy, A. U. and Scott, R. A.," Dynamic stability and Response of a Beam Subjected to a Deflection Dependent Moving Load, "Journal of Vibration, Acoustics, Stress, and Reliability in Design, Vol. 109, pp. 361-365 (1987).

5. Païdoussis, M. P. and Issid, N. T., " Dynamic stability of pipes conveying fluid, "Journal of Sound and Vibration, Vol. 33, pp. 267-294 (1974).

6. Chen, W. H. and Fan, C. N., "Stability Analysis with Lumped Mass and Friction effects in Elastically Supported Pipes Conveying Fluid, "Journal of Sound and Vibration, Vol. 119, pp. 429-442 (1987).

7. Païdoussis, M. P., " Flow-induced Instabilities of Cylindrical Structures," Applied Mechanics Review, Vol.40, No. 2, pp. 163-175 (1987).

8. Frahm, H., " Device for Damping Vibration of Bodies," US patent No. 989958, 30 Oct. (1909).

9. Den Hartog, J.P., Mechanical Vibration, 5th edition, Dover, New York (1985).

10. Young D., "Theory of dynamic absorbers for beams, "Proceedings of the First U.S. National Congress of Applied Mechanical, pp. 91-96 (1952).

11. Jacquot, R.G., " Optimal dynamic vibration absorbers for general beam systems, "Journal of Sound and Vibration, Vol.60, pp. 535-542 (1978).

12. Weaver, W., JR., Timoshenko, S.P. and Young, D.H., Vibration Problems in Engineering, 5th edition, John Wiley and Sons, Inc. (1990).

13. Lin, Y.-H. and Cho, C.-H., "Vibration Reduction for an Elastic Beam Subjected to Multiple Moving Loads, "submitted to Chinese Journal of Applied Mechanics (1992). 